\title{
ECA-immunogenicity of Proteus mirabilis strains
}

\section{Katarzyna Anna Duda ${ }^{1}$, Katarzyna Teresa Duda ${ }^{1}$, Agnieszka Beczała ${ }^{1}$, Katarzyna Kasperkiewicz ${ }^{1}$, Joanna Radziejewska-Lebrecht ${ }^{1}$ and Mikael Skurnik ${ }^{2}$}

${ }^{1}$ Department of Microbiology, Faculty of Biology and Environment Protection, University of Silesia, Katowice, Poland

${ }^{2}$ The Haartman Institute, University of Helsinki and Helsinki University Central Hospital Laboratory Diagnostics, Helsinki, Finland

Received: 2008.05.21, Accepted: 2008.12.19, Published online: 2009.03.31

(C) L. Hirszfeld Institute of Immunology and Experimental Therapy, Wrocław, Poland 2009

\begin{abstract}
Introduction: Bacteria of the genus Proteus are opportunistic pathogens and cause mainly urinary tract infections. They also play a role in the pathogenesis of reactive arthritis (RA). Patients suffering from Yersinia-triggered RA often carry high titers of antibodies specific to enterobacterial common antigen (ECA). The immunogenicity of ECA has not received much attention thus far and studies have focused mainly on the ECA of Escherichia coli and Yersinia enterocolitica. In this paper the ECA-immunogenicity of Proteus mirabilis is elucidated using two wild-type strains (S1959 and O28) as well as their rough (R) derivative strains R110/1959, which expresses lipopolysaccharide (LPS) with a full core, and R4/O28, which expresses LPS with only an inner core.

Materials and Methods: Rabbit polyclonal antisera were produced by immunization with boiled suspensions of the four $P$. mirabilis strains. The antisera were tested for the presence of antibodies specific to ECA by Western blotting using glycerophospholipid-linked ECA (ECA PG $_{\text {}}$ of Salmonella montevideo as antigen. Lipopolysaccharide (LPS) was isolated from the four strains by the hot phenol/water procedure in which $\mathrm{ECA}_{\mathrm{PG}}$ is co-extracted with LPS and by the

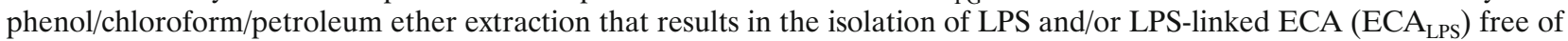
$\mathrm{ECA}_{\mathrm{PG}}$. The LPS preparations were tested for the presence of ECA by Western blotting using ECA-specific antibodies.

Results: The results demonstrated that all four $P$. mirabilis strains were ECA immunogenic. The rabbit antisera immunized by the four strains all contained ECA-specific antibodies. Analysis of the LPS preparations demonstrated that the P. mirabilis wild-type strains O28 and S1959 and the Ra mutant strain R110/1959 expressed $\mathrm{ECA}_{\mathrm{LPS}}$, suggesting that it induced the antiECA antibody responses. Only the presence of $\mathrm{ECA}_{\mathrm{PG}}$ could be demonstrated in the Rc mutant strain $\mathrm{R} 4 / \mathrm{O} 28$.

Conclusions: These results therefore suggest that, similar to E. coli, LPS with a full core is also required as the acceptor of ECA for P. mirabilis strains to produce $\mathrm{ECA}_{\mathrm{LPS}}$. Since $\mathrm{ECA}_{\mathrm{PG}}$ is not immunogenic unless combined with some proteins, it is likely that $\mathrm{ECA}_{\mathrm{PG}}$-protein complexes formed during the intravenous immunization with the Rc mutant strain R4/O28.
\end{abstract}

Key words: Proteus mirabilis, S and R strains, enterobacterial common antigen, lipopolysaccharide, ECA immunogenicity.

Abbreviations: ECA - enterobacterial common antigen, LPS - lipopolysaccharide, PAGE - polyacrylamide gel electrophoresis, $\mathrm{PCP}$ - phenol/chloroform/petroleum ether procedure, $\mathrm{Ph} / \mathrm{W}$ - phenol/water procedure.

Corresponding author: Mikael Skurnik, Ph.D., Professor of Bacteriology, Infection Biology Research Program, Haartman Institute, Department of Bacteriology and Immunology, P.O. Box 21 (Haartmaninkatu 3), FIN-00014 University of Helsinki, Helsinki, Finland, e-mail: mikael.skurnik@helsinki.fi

\section{INTRODUCTION}

The genus Proteus contains five named species: P. mirabilis, $P$. penneri, $P$. vulgaris, $P$. myxofaciens, and $P$. hauseri, and three unnamed genomospecies 4,5 , and 6 (O’Hara et al. 2000a; O'Hara et al. 2000b; Różalski 2002). These Gram-negative rods are widespread in the environment, for example in soil, water, and sewage, and, with the exception of $P$. myxofaciens, are common human intestinal and urinary tract pathogens (Różalski et al. 1997). Under favorable conditions, Proteus infections may lead to serious complications, such as acute or chronic pyelonephritis, the formation of bladder and kidney stones, and catheter obstruction (Różalski 2002). Evidence was recently presented that $P$. mirabilis is an etiopathogenic agent in rheumatoid arthritis, a chronic inflammatory polyarthritic disease (Rashid and Ebringer 2007).

Several virulence factors have been identified in Proteus strains. The most important are fimbriae-medi- 
ated adherence, glycocalyx formation, lipopolysaccharide (LPS), and probably also enterobacterial common antigen (ECA). Interestingly, antibodies against ECA are present in the serum of patients suffering from reactive arthritis (Granfors et al. 1989; Rastawicki 2007).

ECA, a cell surface lipoglycan, is present in all bacteria of the family Enterobacteriaceae, with the exception of the plant pathogenic bacterium Erwinia chrysanthemi (Kuhn et al. 1987; Rinno et al. 1980). ECA is a heteropolymer built of $\rightarrow 3)-\alpha-D-F u c p 4 N A c-(1 \rightarrow 4)-\beta$-D-Man $p$ NAcA- $(1 \rightarrow 4)-\alpha-D-G l c p N A c-(1 \rightarrow$ trisaccharide repeating units (Lugowski et al. 1983). It occurs in three structurally different forms: (i) $\mathrm{ECA}_{\mathrm{PG}}$, which is anchored in the outer membrane of the bacterial cell wall via L-glycerophospholipid, (ii) $\mathrm{ECA}_{\mathrm{LPS}}$, in which lipid A functions as a membrane-anchor and to which ECA is linked via the core oligosaccharide, and (iii) $\mathrm{ECA}_{\mathrm{CYC}}$, which is deprived of a lipid carrier and is located in the periplasm (Kajimura et al. 2005; Kuhn et al. 1988). The $\mathrm{ECA}_{\mathrm{PG}}$ form is not immunogenic unless bound to a protein, which could occur in animal serum after intravenous injection. $\mathrm{ECA}_{\mathrm{LPS}}$, in contrast, is immunogenic. Presence of $\mathrm{ECA}_{\mathrm{LPS}}$ has been demonstrated in $E$. coli Ra mutants possessing the full core type of coli R1, R4, and K-12 in their LPS (Kuhn et al. 1988) and also in $E$. coli Ra mutants with the full core type of coli R2 or R3 (Kasperkiewicz et al. 2004). We have also shown that Yersinia enterocolitica $\mathrm{O}: 3 \mathrm{R}$ mutants with a full core (chemotype $\mathrm{Ra}$ ) as well as those with a truncated outer core or only an inner core (chemotype Rc) in their LPS are ECA immunogenic. In all of them, ECA was linked to the inner core of the LPS (Duda 2007a; Duda 2007b; Radziejewska-Lebrecht et al. 2003). Moreover, the $Y$. enterocolitica O:3 wild-type strain Ye75S was ECA immunogenic in rabbits and contained O-chain polysaccharide as well as ECA bound to the inner core of its LPS (Radziejewska-Lebrecht et al. 1998). In this study we wanted to examine whether $\mathrm{ECA}_{\mathrm{LPS}}$ was also present in Proteus mirabilis, another species of Enterobacteriaceae.

\section{MATERIALS AND METHODS}

\section{Bacterial strains}

P. mirabilis strains O28 (wild type), S1959 (wild type), R4/O28 (rough, Rc chemotype), and R110/1959 (rough, Ra chemotype), described earlier (Radziejewska-Lebrecht et al. 1980; Radziejewska-Lebrecht and Mayer 1989; Vinogradov and Radziejewska-Lebrecht 2000; Vinogradov et al. 2000), were obtained from the collection of the Institute of Microbiology and Immunology in Łódź.

\section{Antigens and serological assays}

LPS preparations (LPS $\mathrm{Ph} / \mathrm{W}$ ) of $P$. mirabilis $\mathrm{O} 28$ (Vinogradov and Radziejewska-Lebrecht 2000) and S1959 (Vinogradov et al. 2000) were obtained by the hot phenol/water procedure of Westphal (Westphal and Jann 1965). LPS $\mathrm{Ph} / \mathrm{W}$ was then subjected to the phenol/chloroform/light petroleum (PCP) procedure of Galanos (Galanos et al. 1969) to obtain LPS PCP. Only the LPS PCP preparation was obtained from the Ra mutant strain R110/1959 by straight PCP extraction of the dry bacterial mass (Radziejewska-Lebrecht and Mayer 1989). For the Rc mutant strain R4/O28, both the LPS $\mathrm{Ph} / \mathrm{W}$ and LPS PCP preparations were acquired directly from dry bacterial mass (Radziejewska-Lebrecht et al. 1980; Radziejewska-Lebrecht et al. 1988). The LPS Ph/W preparations may contain $\mathrm{ECA}_{\mathrm{PG}}$, which co-extracts with LPS in the hot phenol/water procedure, whereas LPS PCP preparations are deprived of ECA $\mathrm{EG}_{\mathrm{PG}}$

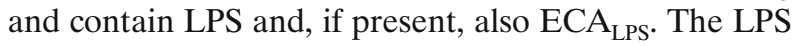
preparations and the control $\mathrm{ECA}_{\mathrm{PG}}$ preparation from S. montevideo SH94 (kind gift from Drs. H. Mayer and H.-M. Kuhn, Max-Planck-Institute, Freiburg, Germany) were used as antigens in SDS/polyacrylamide gel electrophoresis (SDS/PAGE) and Western blotting as described (Radziejewska-Lebrecht et al. 2003). The experiments were repeated five times and representative results are shown in the figures.

\section{Antibodies and antisera}

Monoclonal antibody specific to ECA, mAb 898, recognizing the Man $p$ NAcA epitope (Meier-Dieter et al. 1989) was a kind gift from Prof. D. Bitter-Suermann (Institute of Medical Microbiology, Hannover, Germany).

The monovalent antiserum against the ECA of E. coli $\mathrm{O} 14: \mathrm{K} 7$ was prepared by the adsorption of rabbit antiserum against E. coli $\mathrm{O} 14: \mathrm{K} 7$ by isogenic ECA-negative mutant bacteria. The antiserum, enriched in anti-ECA antibodies, was received from Dr. H. Mayer from the Max-Planck-Institute, Freiburg, Germany (Männel and Mayer 1978). Alkaline phosphatase-conjugated goat anti-mouse and goat anti-rabbit immunoglobulin antibodies (D0486 and D0487, respectively, DAKO) were used in a 1:2000 dilution as the secondary antibodies in Western blotting.

Polyclonal antisera against $P$. mirabilis strains $\mathrm{O} 28$, S1959, and R110/1959 as well as antiserum against Staphylococcus albus (used as a negative control as it lacks cross-reactivity with LPS and ECA, which are typical antigens of Gram-negative bacteria) were obtained by immunization of New Zealand white rabbits with heat-killed bacterial suspensions. Immunization was carried out with the bacterial suspensions $\left(1.5 \times 10^{10} \mathrm{CFU} / \mathrm{ml}\right)$ at doses of $0.25,0.5$, and $1 \mathrm{ml}$ of each over a period of 10 days. The antisera obtained seven days after the last injection were stored at $-20^{\circ} \mathrm{C}$. Polyclonal antiserum against $P$. mirabilis strain R4/O28 was obtained by immunization of New Zealand white rabbits with 500- $\mu$ l doses containing 50, 75, $100,150,200$, and $500 \mu \mathrm{g}$ of lyophilized heat-killed bacterial mass in sterile $0.85 \% \mathrm{NaCl}$ over a period of 51 days. The antiserum obtained seven days after the last injection was stored at $-20^{\circ} \mathrm{C}$. 


\section{RESULTS AND DISCUSSION}

In the present study, two smooth (serogroup O:28 and O:3) and two rough $P$. mirabilis strains were tested for their ECA-immunogenicity. The results (Fig. 1, panel A) demonstrate that ECA-specific antibodies were present in all the tested antisera. The ladder-like banding profile characteristic of ECA was present in the 10 - to $50-\mathrm{kDa}$ region, similar to that in the control reaction with ECA-specific mAb 898 (Fig. 1, panel B). The antisera against the Rc mutant $\mathrm{R} 4 / \mathrm{O} 28$ and the $\mathrm{Ra}$ mutant R110/1959 (the ladder-like profile for this sample is only faintly visible) contained higher titers of anti-ECA antibodies than the antisera against the wild-type strains O28 and S1959. Although there is a possibility of variation among individual rabbits, it is likely that the differences in titers are due to small amounts of immunogenic ECA in the latter bacteria (see below) or to the possibility that in the wild-type strains the ECA chains could be masked by the abundant O-polysaccharides chains on the bacterial cell wall. However, the latter alternative might not be valid as the bacteria are most likely degraded and processed by rabbit antigenpresenting cells during immunization. Acker et al. reported that on whole bacteria, ECA is better exposed on the cell surface of Rc mutant Ye75R bacteria than on the parental S strain Ye75S bacteria (Acker et al. 1981).

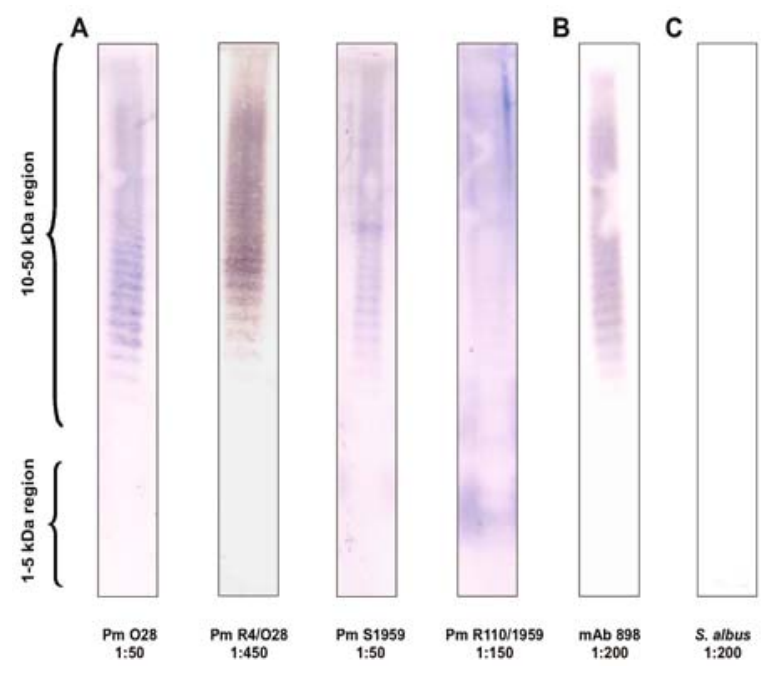

Fig. 1. P. mirabilis-immunized rabbit sera contain ECA-specific antibodies detected by Western blotting. The $\mathrm{ECA}_{\mathrm{PG}}$ of $S$. montevideo $(2 \mu \mathrm{g}$ per lane) was used as the antigen. Panel A. Antisera from rabbits immunized with $P$. mirabilis strains $\mathrm{O} 28$, R4/O28, S1959, and R110/1959. Panel B. ECA-specific monoclonal antibody mAb 898 (positive control). Panel C. Antiserum from rabbit immunized with Staphylococcus albus (negative control). The dilutions of the antisera at which the reactions were carried out are indicated below the blots.

ECA-immunogenicity was previously demonstrated for $E$. coli and $Y$. enterocolitica O:3 strains (Duda 2007b; Kuhn et al. 1988; Radziejewska-Lebrecht et al.
1998; Radziejewska-Lebrecht et al. 2003). We showed above that all the tested $P$. mirabilis strains were also ECA immunogenic and that this was independent of the core structure, similarly to the Yersinia strains. Subsequently, we wanted to elucidate whether the observed ECA-immunogenicity was due to $\mathrm{ECA}_{\mathrm{LPS}}$ or $\mathrm{ECA}_{\mathrm{PG}}$. In the immunoblot analysis developed with ECA-specific mAb 898 (Fig. 2, panel A), all the LPS $\mathrm{Ph} / \mathrm{W}$ preparations from the $P$. mirabilis strains $\mathrm{O} 28$, $\mathrm{R} 4 / \mathrm{O} 28$, and S1959 reacted positively in the high-molecular-mass region, indicating the presence of ECA $\left(\mathrm{ECA}_{\mathrm{PG}}\right.$ and/or $\left.\mathrm{ECA}_{\mathrm{LPS}}\right)$, similarly to the control reaction with $\mathrm{ECA}_{\mathrm{PG}}$. The strongest reaction was observed for the $\mathrm{LPS} \mathrm{Ph} / \mathrm{W}$ of the Rc mutant R4/O28 and the weakest for the LPSs of S1959 and R110/1959 (Fig. 2, panel A). A weak reaction could be detected on the membrane, but it is not visible in the scanned picture; the locations of the stained bands are indicated by braces. In the case of the LPS PCP preparation of the $\mathrm{Rc}$ mutant $\mathrm{R} 4 / \mathrm{O} 28$, no reaction with $\mathrm{mAb} 898$ was observed; however, it was present in the LPS PCP of the parental strain O28. The gel was silver-stained after transfer of the samples to the nitrocellulose membrane (Fig. 2, panel B) and this confirmed the proper loading of the samples onto the gel. At the same time it indicated that the lack of reactivity of the R4/O28 LPS PCP with $\mathrm{mAb} 898$ was likely due to the absence of $\mathrm{ECA}_{\mathrm{LPS}}$ in this preparation. Of note, ECA preparations cannot be silver-stained as there are no vicinal hydroxyl groups in the ECA repeat unit required for the oxidation step in the staining; thus it is not visible in Fig. 2, panel B.

In conclusion, the obtained results suggest that the ECA-immunogenicity of the smooth strains $\mathrm{O} 28$ and S1959 and of the Ra mutant R110/1959 was due to the presence of $\mathrm{ECA}_{\mathrm{LPS}}$, whereas that of the Rc mutant $\mathrm{R} 4 / \mathrm{O} 28$ was due to the presence of $\mathrm{ECA}_{\mathrm{PG}}$. As mentioned, the latter co-extracts with LPS in the $\mathrm{Ph} / \mathrm{W}$ procedure.

Our previous studies on $\mathrm{R}$ mutants of $Y$. enterocolitica O:3 showed that monovalent antiserum against $E$. coli O14:K7 enriched with ECA-specific antibodies was a more sensitive tool in the detection of ECA than $\mathrm{mAb}$ 898 , perhaps reflecting a lower affinity of the monoclonal antibody (Duda 2007a). In line with this, the immunostainings with the monovalent antiserum (Fig. 3, panel A) were stronger than with $\mathrm{mAb} 898$ (Fig. 2, panel A). All the $P$. mirabilis LPS preparations gave positive reactions in the 10 - to $50-\mathrm{kDa}$ region corresponding to ECA compared with the control reaction with $\mathrm{ECA}_{\mathrm{PG}}$ (Fig. 3, panel A), with the exception of the LPS PCP of R4/O28. As above, the gel was silver-stained after transfer of the samples to the nitrocellulose membrane (Fig. 3, panel B) and this confirmed the proper loading of the samples onto the gel, including that of the R4/O28 LPS PCP. As mentioned above, the ECA-immunogenicity of this Rc mutant was most likely due to $\mathrm{ECA}_{\mathrm{PG}}$. $\mathrm{ECA}_{\mathrm{PG}}$ has been reported to be a very poor immunogen; however, its immunogenicity would increase if it were covalently bound or associated with 


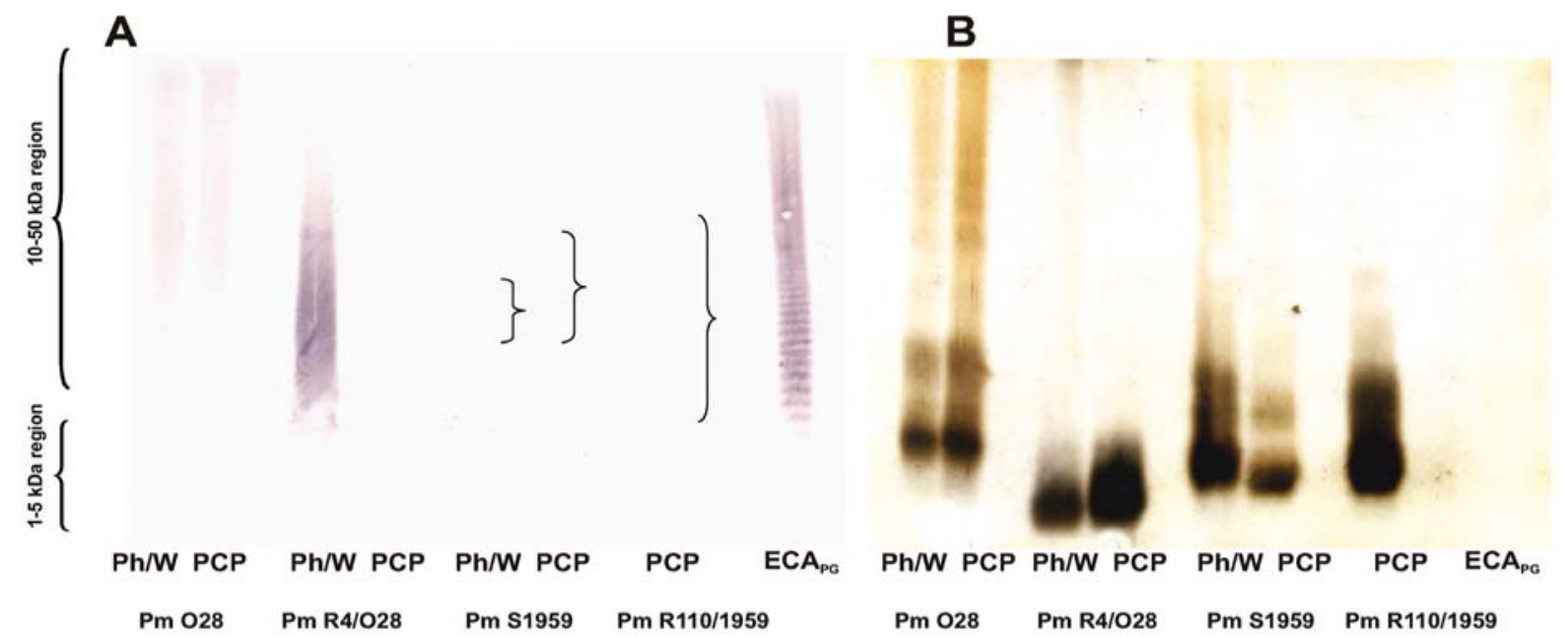

Fig. 2. Detection of ECA in the LPS preparations of $P$. mirabilis strains. Panel A. Western blotting using ECA-specific mAb 898 at a 1:200 dilution. Panel B. Silver-stained gel after transfer of the samples to the PVDF membrane. The LPS Ph/W and LPS PCP preparations (15 $\mu \mathrm{g}$ per lane) of $P$. mirabilis strains O28, R4/O28, S1959, and R110/1959 and $\mathrm{ECA}_{\mathrm{PG}}$ of S. montevideo (positive control, $2 \mu \mathrm{g}$ per lane) were analyzed as indicated below the images.

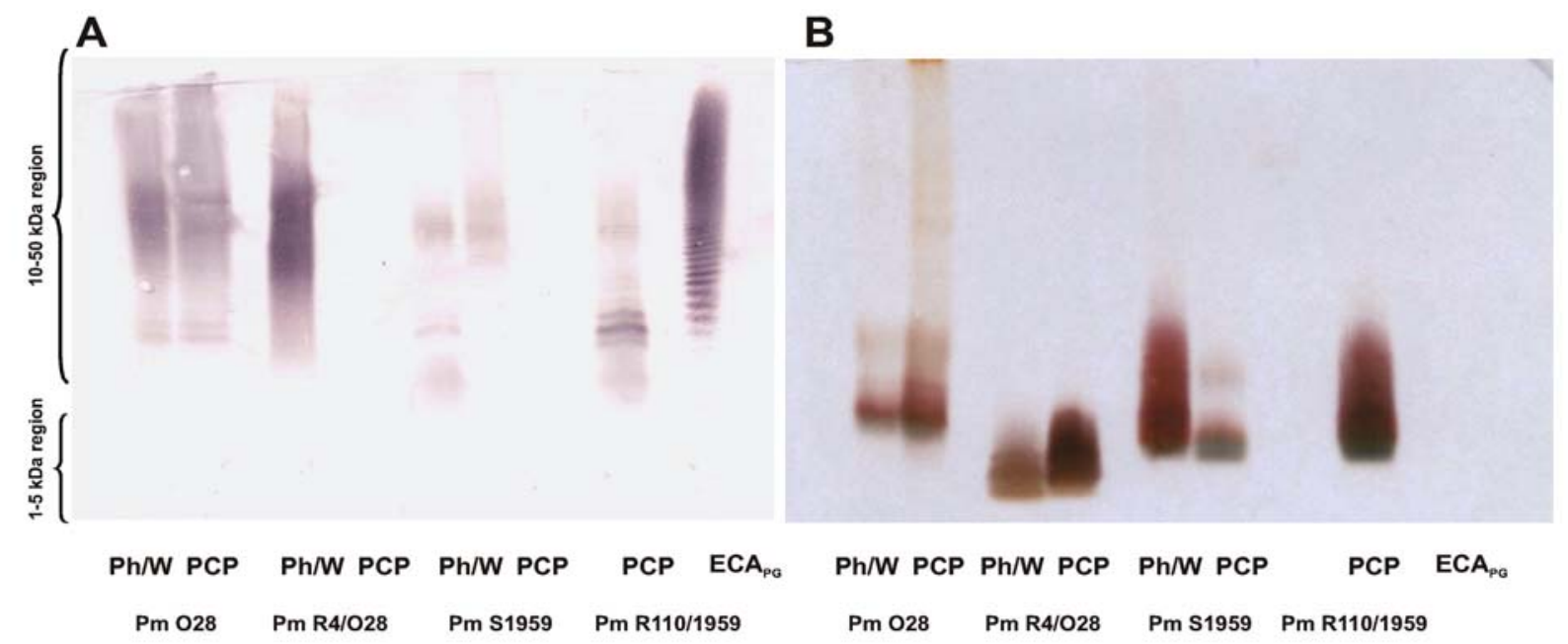

Fig. 3. Detection of ECA in the LPS preparations of $P$. mirabilis strains. Panel A. Western blotting using 1:200 diluted monospecific anti-E. coli O14:K7 antiserum enriched in ECA-specific antibodies. Panel B. Silver-stained gel after transfer of the samples to the PVDF membrane. The LPS Ph/W and LPS PCP preparations (15 $\mu$ g per lane) of $P$. mirabilis strains O28, R4/O28, S1959, and R110/1959 and ECA ${ }_{\mathrm{PG}}$ of $S$. montevideo (positive control, $2 \mu \mathrm{g}$ per lane) were analyzed as indicated below the images.

a carrier, for example proteins (Kuhn et al. 1988), and this is the likely explanation for the ECA immunogenicity of the Rc mutant R4/O28.

The expression of $\mathrm{ECA}_{\mathrm{LPS}}$ by the $\mathrm{Ra}$ mutant R110/1959 with a full core region and not by the Rc mutant $\mathrm{R} 4 / \mathrm{O} 28$ with a truncated core region suggests that in $P$. mirabilis, similarly to E. coli, the complete core region of LPS is a prerequisite for the linking of ECA to LPS. This conclusion is additionally supported by the following two observations. (i) The presence in the 1- to $5-\mathrm{kDa}$ region of the membrane of the two clearly sepa- rated ECA-positive bands in the LPS PCP preparation of strain O28 and several similar bands in the LPS PCP of the Ra mutant R110/1959; these bands correspond to the migration of full core molecules (Fig. 3, panel A). (ii) The lack of ECA-specific immunostaining in the 1to 5-kDa region of the LPS PCP preparation of the Rc mutant $\mathrm{R} 4 / \mathrm{O} 28$, which possesses only the inner core in its LPS.

In contrast to previous observations (Kuhn et al. 1988), it is very likely that $\mathrm{ECA}_{\mathrm{LPS}}$ occurs abundantly in nature and one can envision that in wild-type strains 
under certain environmental conditions, a higher proportion of LPS molecules could be unsubstituted by O-polysaccharide and they could then become ECA-substituted. Our results therefore warrant further studies to find out whether other members of Enterobacteriaceae in addition to E. coli, $Y$. enterocolitica, and $P$. mirabilis express ECA-immunogenic ECA $_{\mathrm{LPS}}$.

Acknowledgment: We wish to thank Prof. Antoni Różalski (Łódź, Poland) for the gift of rabbit anti-Proteus antisera.

\section{REFERENCES}

Acker G, Knapp W, Wartenberg K et al (1981) Localization of enterobacterial common antigen in Yersinia enterocolitica by the immunoferritin technique. J Bacteriol 147:602-611

Duda KA (2007a) Immunochemical studies on lipopolysaccharides from $\mathrm{R}$ mutants Yersinia enterocolitica $\mathrm{O}: 3$. Ph D Thesis. University of Silesia, Katowice, Poland and Research Center Borstel, Borstel, Germany

Duda KT (2007b) Reactivity of polyclonal antisera against $\mathrm{R}$ mutants of Yersinia enterocolitica O:3. Ph D Thesis. University of Silesia, Katowice, Poland and The Haartman Institute, University of Helsinki, Helsinki, Finland

Galanos C, Lüderitz O, Westphal O (1969) A new method for the extraction of $\mathrm{R}$ lipopolysaccharides. Eur $\mathrm{J}$ Biochem 9:245-249

Granfors K, Jalkanen S, von Essen R et al (1989) Yersinia antigens in synovial-fluid cells from patients with reactive arthritis. N Engl J Med 320:216-221

Kajimura J, Rahman A, Rick PD (2005) Assembly of cyclic enterobacterial common antigen in Escherichia coli K-12. J Bacteriol 187:6917-6927

Kasperkiewicz K, Skurnik M, Brade L et al (2004) Monoclonal antibodies specific for lipid A and ECA as tools in characterization of lipopolysaccharides from enterobacterial R mutants. $3^{\text {rd }}$ German-Polish-Russian Meeting on Bacterial Carbohydrates, October 6-9, Wrocław, Poland. Abstract P10

Kuhn HM, Basu S, Mayer H (1987) Comparison of enterobacterial common antigen from different species by serological techniques. Eur J Biochem 162:69-74

Kuhn HM, Meier-Dieter U, Mayer H (1988) ECA, the enterobacterial common antigen. FEMS Microbiol Rev 4:195-222

Ługowski C, Romanowska E, Kenne L et al (1983) Identification of trisaccharide repeating unit in the enterobacterial common antigen. Carbohydr Res 118:173-181

Männel D, Mayer H (1978) Isolation and chemical characterization of the enterobacterial common antigen. Eur J Biochem $86: 361-370$

Meier-Dieter U, Acker G, Mayer H (1989) Detection of enterobacterial common antigen on bacterial cell surfaces by colony-immunoblotting: effect of its linkage to lipopolysaccharide. FEMS Microbiol Lett 50:215-220
O’Hara CM, Brenner FW, Miller JM (2000a) Classification, identification, and clinical significance of Proteus, Providencia, and Morganella. Clin Microbiol Rev 13:534-546

O'Hara CM, Brenner FW, Streigerwalt AG (2000b) Classification of Proteus vulgaris biogroup 3 with recognition of Proteus hauserii sp. nov. nom. rev. and unnamed Proteus genomospecies 4, 5 and 6. Int $\mathrm{J}$ Syst Evol Microbiol 50:1869-1875

Radziejewska-Lebrecht J, Bhat UR, Brade H et al (1988) Structural studies on the core and lipid A region of a 4-amino-L-arabinose-lacking Rc-type mutant of Proteus mirabilis. Eur J Biochem 172:535-541

Radziejewska-Lebrecht J, Feige U, Jensen M et al (1980) Structural studies on the glucose-heptose region of the Proteus mirabilis R core. Eur J Biochem 107:31-38

Radziejewska-Lebrecht J, Kasperkiewicz K, Skurnik M et al (2003) ECA-antibodies in antisera against $\mathrm{R}$ mutants of Yersinia enterocolitica O:3. Adv Exp Med Biol 529:215-218

Radziejewska-Lebrecht J, Mayer H (1989) The core region of Proteus mirabilis R110/1959 lipopolysaccharide. Eur J Biochem 183:573-581

Radziejewska-Lebrecht J, Skurnik M, Shashkov AS et al (1998) Immunochemical studies on R mutants of Yersinia enterocolitica O:3. Acta Biochim Pol 45:1011-1019

Rashid T, Ebringer A (2007) Rheumatoid arthritis is linked to Proteus - the evidence. Clin Rheumatol 26:036-1043

Rastawicki W (2007) Humoral response to selected antigens of Yersinia enterocolitica and Yersinia pseudotuberculosis in the course of yersiniosis in humans. I. Occurrence of antibodies to enterobacterial common antigen (ECA). Med Dosw Microbiol 59:93-102

Rinno I, Golecki JR, Mayer H (1980) Localization of enterobacterial common antigen: Immunogenic and nonimmunogenic entrobacterial common antigen-containing Escherichia coli. J Bacteriol 141:814-821

Różalski A (2002) Molecular basis of the pathogenicity of Proteus bacteria. Adv Clin Exp Med 11:3-18

Różalski A, Sidorczyk Z, Kotełko K (1997) Potential virulence factors of Proteus bacilli. Microbiol Mol Biol Rev 61:65-89.

Vinogradov E, Radziejewska-Lebrecht J (2000) The structure of the carbohydrate backbone of the core-lipid A region of the lipopolysaccharide from Proteus mirabilis serotype O28. Carbohydr Res 329:351-357

Vinogradov E, Radziejewska-Lebrecht J, Kaca W (2000) The structure of the carbohydrate backbone of core-lipid A region of the lipopolysaccharides from Proteus mirabilis wild-type strain S1959 (serotype O3) and its Ra mutant R110/1959. Eur J Biochem 267:262-269

Westphal O, Jann K (1965) Bacterial lipopolysaccharide extraction with phenol-water and further application of procedure. Met Carbohydr Chem 5:83-91 